\title{
Caracas: un intento frustrado de continuidad de las Bienales Hispanoamericanas de arte (II)
}

\author{
Miguel Cabanas Bravo
}

\section{LA BIENAL FRUSTRADA}

La participacion venezolana en cada una de las ediciones de la Bienal Hispanoamericana, aunque rodeada de electicismo e indefiniciones, no habia variado mucho en ninguna de ellas, lo que estaba en consonancia con la misma vaguedad en la consideración y actuación oficial de Venezuela respecto al certamen, pese a que siempre se hubiera conseguido la presencia oficial de este país en sus torneos.

El hecho hace más interesante y llamativa la intención de celebrar la IV Bienal Hispanoamericana en Caracas, aunque claro está que se estaban priorizando nuevamente y de manera aun más evidente los intereses politico-económicos sobre los de indole artística. Esta nueva actuación hay que considerar que se instalaba ya en un nuevo momento de la política española, es decir, el que comenzaba a principios de 1957 con la llegada de un nuevo Gobierno - quinto Gobierno del régimen o "Gobierno de la Estabilización»-, que reemplazaba a Alberto Martin Artajo en la cartera de Asuntos Exteriores por Fernando Maria Castiella, lo que también tendria como consecuencia la sustitución de Alfredo Sánchez Bella -quien se incorporaba a comienzos de este año a su nuevo cargo de embajador de España en Santo Domingo- por Blas Piñar como director del Instituto de Cultura Hispánica.

No obstante, todavia en los momentos en que Sánchez Bella dirigía este Instituto, se emprendió pronto la actividad tendente a lograr celebrar una nueva edición del certamen en América, comenzando por la redacción de una "Memoria y Memorandum» sobre las tres Bienales Hispanoamericanas celebradas y las posibilidades de realizar la IV Bienal en Caracas, presentada por la Secretaría Permanente de la Bienal Hispanoamericana de Arte con la intención evidente de que fuera conocida por el Gobierno venezolano. El largo informe, dividido en tres epigrafes 
y profundamente retórico e insistente en destacar la estrecha vinculación e identidad común de los pueblos hispanos, comenzaba por señalar en el primero - «Alcance y significación de la Bienal»- que ésta era una institución que no se limitaba "al carácter transitorio que pudiera adquirir en cualquiera de sus versiones", sino que: “Nace de la certidumbre de que los diversos artes de los pueblos nispánicos están poseídos de una genealogia y de unas aspiraciones comunes. Intenta poner de manifiesto lo que hay, efectivamente, de idéntico en su rica diversidad. Intenta asimismo que esta identidad se haga patente a todo artista de la comunidad de pueblos hispánicos. Finalmente, pretende informar de una manera periódica del estado de las artes en cada uno de esos pueblos". Su importancia, pues, rebasaba con creces - proseguía el documento- «el estrecho límite de las catalogaciones artísticas, para entrar de lleno en la realización ideal de una cultura patrimonio de la mayor y la mejor comunidad de pueblos que existe en el presente. Alcanza, incluso, en el mejor sentido de la palabra a la realización de una gran politica, entendiendo•por tal la posibilidad del entendimiento entre los hombres».

Se añadía también que, la Bienal Hispanoamericana, asimismo venía a poner de manifiesto "un hecho que se hizo certeza en los mejores hombres de la estirpe: el de la originalidad de la cultura y de las artes hispáni cas. Estamos seguros de que vivimos los tiempos iniciales en que la cultura hispánica, y muy concretamente la cultura de los pueblos de Hispanoamérica, puede influir con eficacia en el mundo. En el caso concreto de las artes plásticas pretende ponerlo de manifiesto esa institución que es la Bienal». Ello hacia «natural» que se diferenciara de otras organizaciones del mismo tipo, como la Bienal de Venecia y la de Sao Paulo, pues la Hispanoamericana trataba de "encontrar la unidad de lo diverso" en los pueblos hispánicos al mismo tiempo que trataba de "diferenciar las caracteristicas de esta unidad en todo el ámbito internacional», o sea, pretendia “una universalización del arte hispánico», ya que la «la historia del arte de nuestro siglo se ha realizado esencialmente desde Paris y en último extremo desde Europa, con las lógicas consecuencias de extremada parcialidad», que había hecho que tal originalidad de la cultura y el arte hispánico fuera «incluso desconocida para nosotros mismos».

En cuanto a su criterio artístico, la Bienal Hispanoamericana se definía como "un organismo absolutamente ecléctico en cuanto a la apreciación de la obra de arte, según la temática o el estilo conceptual», es decir, no pretendía "delimitar el campo de las tendencias", aunque poseia "un estilo que subyace por debajo de la amplitud de su criterio", caracterizado «por la vitalización y reconocimiento de un arte con validez de actualidad histórica». Por otro lado, señalaba, en cuanto a su adscripción geográfica, 
que ei hecho de que, "hasta el presente, haya caido sobre organismos españoles o asentados en España la mayor responsabilidad organizativa no significa en modo alguno que la Bienal sea una institución española. Pretende, eso sí, en la medida de lo posible, demostrar que en el terreno del arte al menos España puede llegar a ser un país americano. Desde el punto de vista exclusivamente español esta es una de las aspiraciones, pues entretejida en toda su organización está la firme creencia de que la próxima escuela de validez universal no será ya la escuela de París, sino la escuela de América. Se trata, pues, de un organismo supranacional perteneciente por igual a todos los países hispánicos".

Continuaba después la "Memoria-Memorandum", en el segundo de sus epigrafes, "Origen e historia», rememorando lo que habian sido las tres Bienales celebradas anteriormente, para lo que se ofrecian algunos datos y se hacian resaltar algunas circunstancias incidentes en mostrar su singularidad y trascendencia '. Finalmente, en el último de estos apartados, "Factores y conveniencia de la Bienal en Caracas", se exponia, en elogiosos términos, las razones que hacian deseable la celebración de

Ası. aparte de los datos de celebracion, cuantia de los premios, numero de paises, artistas y obras concursantes, eic.. se destacaban hechos sobre la I Bienal, como que en la inauguración el ininistro de Educación Nacional pronunció un discurso que "sıgnificaba toda una definicion política sobre el arte y el Estado: “la postura exacta del Estado ante el arte es la de maximo respeto para la autonomia creadora del artista. Lo unico que cabe exigir al artista es autenticidad y al propio tiempo hay que estimularle para vincular su trabajo al tiempo en que vive y a su tiena". Definición que, digámoslo de paso, continua siendo la consigna de todas nuestras Bienales". O se señalaba: "De la significacion de esta primera Bienal en el ambiente español nada puede ofrecer tan cabal idea como la formidable polemica suscitada en todo el ámbito nacional respecto a la apreciación de la obra de arte. En aquel tiempo el arte llego a ser tan popular en España como las manifestaciones deportivas o cinematográficas. Más de medio millón de visitantes tuvo la Bienal en Madrid, y, más tarde, en la antologia llevada a Barcelona, cerca de ochenta mil. De la influencia que la Bienal ha ejercido en el ámbito artístico español da idea el hecho de que la Exposición Nacional de Bellas Artes, tradicionalmente cerrada a todo lo que signifique innovación artistica, ha dado cabida desde entonces a todas las modernas corrientes de la plástican. Sobre la ll Bienal se justificaba: "Es lastima que un sectaria interpretación de las intenciones supranacionales de la Bienal, asociara a esta, en la idea de muchos importantes artistas cubanos, con pretendidos parciales apetitos unllateralmente españoles. Ello motivo una reaccion de tonao patriótico pero de iorcidas consecuencias en el animo de muchos artistas de Cuba que, a la larga, se dieron cuenta do la lalsedad de su posición. Pero la Bienal tuvo que retrasar en álgunos meses su fecha de inauguracion: Y Y, por ultımo, sobre !a III Bienal se destacada que su radicacion en Barcelona, ciudad tan ligada a la evolución de la pintura europea contemporanea, "hizo que el Museo de Arte de Ginebra solicitase una exposicion antologica de la Bienal para celebrarse en aquella ciudad, to que trajo como consecuencia el que se pusiera en el primer plano de la actualidad europea al arte moderno de América. Se lograba asi uno de los fines más especificos de la Bienal Hispanoamericana de Arte, cual es el de incidir en la opinion europea - hasta el momento decisiva en la comprension de las artes plasticas- en el sentido de crear la conciencia de que existe un arte americano de validez universalista". 
la nueva edición del certamen en Caracas, verdadera intención que habia motivado la redacción del escrito. Se decía aquí:

"Muchos son los factores que aconsejan la celebración de la IV Bienal Hispanoamericana de Arte en la ciudad de Caracas, pero vamos a atenernos por el momento al más significativo, al que pudiéramos denominar. resumiendo al máximo, factor geopolitico del arte americano.

Sabido es que, en el presente, el arte que se realiza en América ofrece perspectivas absolutamente distintas. La primera de ellas se deriva del hecho de que gran parte del arte americano del momento, con un evidente sentido mimético, trata de equipararse a la entidad del arte europeo contemporáneo.

Lamentable falla que incapacita para toda originalidad a todo el arte que, por ejemplo, se está realizando actualmente en los Estados Unidos y en Buenos Aires.

La segunda perspectiva se deriva de una pretension de originalidad falsamente entendida. Es el arte que comunmente se realiza en México. Este arte pretende ser original por el tema a tratar y no por la forma de produ. cirse.

Nosotros tenemos la certeza de que el unico arte que en la actualidd se está produciendo en Iberoamérica con posibilidades originalistas está situado en la linea, que coincide precisamente con la de los paises del Caribe, con Caracas como centro natural. En este arte, la originalidad no es una pretensión sino una consecuencia. $Y$ una consecuencia precisamente de todos los elementos étnicos y culturales que integran a los pueblos caribeños. Ocurre, sin embargo, que esta posibilidad de un originalismo americano del que el Caribe sea el centro catalizador es insuficientemente conocido por la joven América, pues el mimetismo europeısta de que hablábamos impide reflexiona: serenamente sobre el problema.

Es necesario, para ello, poner de manifiesto la auténtica valia de muchos jóvenes artistas venezolanos, gentes en quienes el sentido original del arte está tan arraigado que ni siquiera la presencia conturbadora de París ha podido desvirtuarlo. En definitiva, la pretensión de una Bienal en Caracas no es tanto la exaltación de los valores de una pintura nacional venezolana cuanto la exhortación a toda la juventud artistica iberoamericana a retomar las riendas de la originalidad del arte. "s.

A esta actitud afectada de la Secretaria de la Bienal, que venía a asegurar a Caracas el arbitrio en el Caribe del arte americano con "posibilidades originalistas: si hacia valer a sus artistas, se unieron las rápidas gestiones para crear una Comisión Organizadora de la Bienal en Caracas, que segun sugerencia del director del Instituto de Cultura Hispánica tras su

Memorla informativa sobre las tres Bienales Hispanoamericanas de Arte celebradas hasta ahora y memorandum de posibilidades para una IV Bienal, a celebrar en la ciudad de Caracas./ Lo presenta la Secretaria permanente de la Bienal Hispanoamericana de Arte". (s./t., 12 pág.; RGA. ECl, Caja 250, carpeta 6650). 
viaje a Venezuela, upodría estar integrada por Alejandro Pietri, Pedro Vallenilla, Centeno, López Méndez y Armando Barrios" "3.

No obstante, ya durante la dirección del Instituto de Cultura Hispánica por Blas Piñar, un Despacho de finales de febrero de 1957 del embajador de España en Caracas, Manuel Valdés, en el que informaba al canciller de Asuntos Exteriores español de su visita al ministro de Educación venezolano, Dario Parra, para concretar todo lo referente al proyecto de celebración de la IV Bienal en Caracas, daba cuenta de la renuncia venezolana a acogerla según instrucciones del Presidente de la República, así como de la razón - o sea, el que todos los Ministerios debian dedicar su atención y presupuestos a la preparación de las elecciones presidenciales de principios de 1958- y el mantenimiento del ofrecimiento para que en el futuro pudiera celebrarse alguna de las próximas ediciones en Venezuela ".

Sin embargo, y pese a haberse expresado esta renuncia venezolana, la Embajada de España recibía poco después una comunicación de la Dirección de Cultura y Bellas Artes del Ministerio de Educación venezolano, donde señalaba que con fecha del 4 de febrero la Dirección habia recibido una comunicación del Instituto de Cultura Hispánica referente al envio, consignado a esa Embajada, "de seis cajas de cuadros destinados a la III Bienal de Arte Hispanoamericano que habría de celebrarse en Caracas», por lo que, "de acuerdo con instrucciones expresas del ciudadano Ministro y por el hecho de no haber resuelto en definitiva el Gobierno Nacional aceptar como sede a Caracas para efectuar la mencionada Bienal", se informaba que había sido "devuelta a la Compañía de Alfredo Ravard \& Ca. Sucesores, la factura por el costo del flete de dichas cajas, indicándoles que es la Embajada de España la que debe hacer el pago en referencia". Añadia el embajador, a su traslado de esta cornunicación al ministro español, que, como ya había informado, Venezuela no se hacia

Minuta de fecha 3-x/-56 dirigida por Luis Hergueta, secretario técnico del ICH, a Leopoldo Panero, secretario general de la Bienal, trasmitiéndole esta indicación según nota atrasada del director del centro tras su viaje. (RGAECl, Caja 2167, carp. 7199 ).

Decia el embajador: "Este (Dario Parra) me indicó que, sometido el proyecto a S.E. el Presidente de la República, el Primer Magistrado de la nación le ha dado instrucciones renunciando a la aceptación por parte de Venezuela para que la citada exposición tenga lugar en este pals. La razón de ello se debe a que este año comienza el periodo preelectoral ya que las elecciones tendrán lugar a principios de 1958- y todos los Ministerios deben dedicar su atención y presupuestos (salvo to que ya esté comprometido anteriormente) a la preparacion de dichas clecciones presidenciales-. El Ministro de Educación me ha manifestado que lamenta que estas circunstancias impidan el dar acogida a la Bienal en Caracas y que, como no existe otra razón para la renuncia, Venezuela mantiene su ofrecimiento para el futuro y espera que la Comisión Organizadora lo tenga en cuenta para alguna de las próximas exposiciones y entonces este pais podra dar a la Bienal la atencion y ayuda que tan importante manifestacićn artistica hispanoamericana merece". (Despacho $n^{\circ} 66$ fechado: Caracas, 21-1i-57. AMAE, Leg. R-5229, Exp. 67). 
cargo ese año de la proyectada Bienal "que efectivamente no ha pasado nunca de ser un proyecto y por lo tanto es extraño que se haya hecho un envío de cuadros como el que se cita en la anterior comunicación, sin haber contado antes en firme con la aceptación del Gobierno de este país" 5 .

Y es que en España aún se intentaba que la IV Bienal fuera celebrada en Caracas, aunque se retrasara al año siguiente, cuando hubieran pasado las elecciones. En este sentido, con fecha 18 de mayo se dirigía el nuevo ministro español de Asuntos Exteriores, Fernando María Castiella, en su calidad de presidente del Patronato del $1 \mathrm{CH}$, al embajador de Venezuela en España, Simón Becerra, rogándole que pusiera en conocimiento del Gobierno de su país el propósito del español de celebrar en Caracas la IV Bienal Hispanoamericana de Arte, a objeto de recabar el patrocinio del Presidente de la República para la muestra, y haciendo saber que lo más interesante por el momento era saber con antelación si el Gobierno de Venezuela estaría dispuesto a considerar de un modo formal la probabilidad de que el certamen se realizara en Caracas en la primavera del próximo año de $1958^{\circ}$. Aunque, siguiendo la tónica de demoras y dilaciones que caracteriza toda la relación de Venezuela con la Bienal Hispanoamericana, el embajador y luego el Ministerio de Relaciones Exteriores, se retrasarian mucho en dar curso e informar de esta carta, de modo que hasta finales de noviembre de 1957 no se avisaría de la misma al Ministerio de Educación venezolano y hasta diciembre no se consultaria al Presidente de la República?

Pero sobre lo ocurrido en Venezuela respecto a la celebración de la IV Bienal, pronto contó el $\mathrm{ICH}$ con la interpretación y análisis transmitidos por el escritor y agregado cultural español José Luis Acquaroni, en esos

Añadia también el embajador que habia considerado oportuno no hacerse "cargo del pago solicitado, dando las instrucciones necesarias para en su dia, sean devueltas las seis cajas de cuadros a flete debido" (Despacho $n^{\prime} 77$ fechado: Caracas, 28-il-57, que traslada la referida comunicacion de la Dirección de Cultura venezolana a la Embajada española. AMAE, Leg. R-5229, Exp. 67).

Nota $n^{\prime 1} 1.032$ fechada: Madrid 23-v-57 del embajador de Venezuela dirigida ai ministro de Asuntos Exteriores español acusando recibo de su carta $18-v-57$ y reseñando el contenido de la misma de! que informaria a su Gobierno (AMAE, Leg. R-5229, Exp. 67).

Nota $n^{*} 45$ fechada:Madrid 15-1-58 del embajador de Venezuela en España dirigida al ministro de Asuntos Exteriores español, donde le transcribe la nota n' IEB-04889 de 20-XI|-57 que le rabia dirigido el Ministerio de Relaciones Exteriores venezolano, que a su vez era transcriptiva del Oficio n 761 fechado 11-XI1-57 del ministro de Educación venezolano, Dario Parra, que acusaba recibo de la comunicación $n^{\circ}$ IEB-4566 de fecha 29-x|-57 del Ministerio de Relaciones Exteriores -en la que se le informaba de la carta del ministro español expresando el deseo de que Venezuela fuera la próxima sede de la Bienal- y contestaba a la misma tras su entrevista con el Presidente (AMAE, Leg. R-5229, Exp. 67) 
momentos en la Universidad Central de Venezuela, en Caracas, que a mediados de septiembre de 1957 escribia a Alfredo Sánchez Bella, ex-director del $1 \mathrm{CH}$ y embajador de España en la República Dominicana, ante su indignación por to que, sobre la fallida Bienal caraqueña, había comentado a su hermano Miguel Acquaroni y éste habia dado a conocer en el Instituto. Decía en su carta que la información enviada a su hermano provenía de Jesús Antonio Cova y Manuel Felipe Rugeles, habiéndosele lamentado Cova - segundo vicepresidente del Senado-en más de tres ocasiones «de la descabellada propuesta de Bienal formulada por el Gobierno español, y de todo eso de los 900000 Bolívares, el barco fletado, el numeroso cuerpo de secretarios y agregados, etc. Igual versión parecía encargarse de divulgar el Director de Cultura y Bellas Artes, aunque la ligereza o mala fe de éste - filocomunista; dolido con nuestra Embajada porque no se contó con él cuando tu visita del año pasado, época en que estaba temporalmente separado del cargo- se comprende hasta cierto punto; no así la de Cova, en quien esa manifiesta labor de zapa para con la Bienal tiene su antecedente exclusivo - hay que conocer su manera apasionada e impulsiva- en su actual desavenencia con Valdés. Todo muy lamentable, pero real e indiscutible». Pero añadía que no se había conformado con "estas versiones de Cova y Rugeles, apasionadas y feamente intencionales» y habia acudido «a ese hombre ecuánime, sensatisimo, de indudable ascendencia dentro del Ministerio de Educación, buen amigo de España, que es Eduardo Lira Espejo. Lira resultó ser, con toda seguridad, el notario más informado y calificado que tuvo aquel fallido intento de Bienal", trasmitiéndole a titulo confidencial su versión, que relataba asi:

«Según Lira, el error básico resultó de planteamiento. Dificilmente -me decia- podrá darse otra oportunidad de algo tan bien dispuesto para ser aceptado por la parte venezolana como esto de la organización de la Bienal caraqueña. Habia incluso destinado un millón de Bolivares. "Pero por parte de Madrid - son sus palabras- hubo precipitación y falta de tacto". Precipitación - según él- porque en el Ministerio de Educación y en la Presidencia cayó mal el nombramiento de un comisario para la Bienal de Caracas, nombramiento que agencias y periudicos divulgaron a los cuatro vientos, cuando el Gobierno no había aceptado ni en principio la organiza. ción del certamen. $Y$ todavía parece que gustó menos al Ministro, y al propio Presidente, el estilo de la Memoria y Memorandun..

Parece que de ese Memorandun, en primer lugar hizo sonreir todo eso de que "la próxima escuela de arte de validez universal no será ya la escuela de Paris, sino la de América". Luego, lo de "la pretensión de originalidad falsamente entendida del arte que comunmente se realiza en México", empezó ya, francamente, a disgustar. Pareció innecesaria esa negación de valores del arte de un pueblo hermano; arte, por otra parte, "comunmente" reconocido en 
todo el Contınente cuando menos, y del que Hispanoamérica, acertada o erroneamente, se siente orgullosa, por estimarlo - tal vez a falta de otros exponentes, esa es la verdad- bastante representativo. Y empezó a oler a chamusquina, sorprendiendo y disgustando aún más, el "nosotros tenemos la certeza de que el único arte que en realidad se está produciendo en Iberoamérica con posibilidades originales está situado en una linea que coincide precisamente con la de los paises del Caribe, con Caracas como centro natural". Por favor...: si de algo tienen los venezolanos una clara conciencia, una medio clara conciencia, es de su insignificancia cultural. Acá, la palabra cultura obsesiona de tal manera que con ella se anda bautizándolo todo: emisoras de radio, casas comerciales, lineas de transportes urbanos, periódicos de penitenciarias... La verdad es que esto supo a tomadura de pelo. No se puede olvidar - ay, manes de los agregados culturales- que la sicología del venezolano actual es la del nuevo rico, hasta ayer mismo campesino ignorante, siempre con la mosca detrás de la oreja, permanentemente en guardia contra todo halago, que él, naturalmente, entiende encaminado sólo a ablandar el cierre de su bien repleta bolsa. La opinión de Lira Espejo es que por aqui cayó el proyecto de la Bienal - yo creo que exajera, porque puestos a buscar tres pies al gato, cualquier escrito de este tipo puede ser pulverizado-. Que cogieron el rábano por las hojas, eso no se puede dudar. Pero a gentes asi lo más sensato es darle el rábano pelado y mondado, sin hojarascas ni sutilezas.

Naturalmente que, pese a todo, la mala impresión del Memorandun pudo ser ampliamente contrarrestada. Pero la inoperancia e indiferencia - todo hay que decirlo- de dos entidades llamadas a pelear por la Bienal, como eran nuestra Embajada y el Instituto Venezolano de Cultura Hispánica, hicieron perdurar el mal sabor de boca, sin hacer nada por remediarlo... Por parte de unos, hubo un manifiesto interés en crear un ambiente equivoco y contrario a la bienal; por la de otros, no se ha querido mover un dedo para hacer que el problema fuera enfocado en su esencia y totalidad, sin parar mientes en estilos y detalles de planteamientos. Hasta qué punto sería acertado volver sobre la cuestión, esto yo no lo sé. Por otra parte, aqui se habla ya de una IV Bienal en Quito. De todas formas, la visita de Blas Piñar puede ser provechosa en extremo..." ".

Seguramente, pues, además del mal planteamiento que el Instituto hizo del proyecto de la Bienal y el desinterés y falta de eficacia de las instituciones españolas en Caracas más implicadas con el tema, hubo también en el mundo oficial venezolano pasividad, vacilaciones, suceptibilidades y suspicacias sobre la organización de esta muestra. Sin embargo, el Instituto de Cultura Hispánica no estaba dispuesto a perder la ocasión venezolana, por lo que el propio presidente de la institución viajó

Carta de José Luis Acquaronı a Alfredo Sanchez Bella fechada 15-Ix-57. El propio J.A.Acquaroni enviaba copia de la misma junto a su carla de fecha $20-x-57$ a Luis Hergueta, secretario técnico del ICH, para que el Instituto supiera de "la cuestión" al igual que Sánchez Bella (Ambas cartas en RGAECl. Caja 2167, carp. 7199). 
en octubre a Caracas para plantear nuevamente la celebración de la IV Bienal. "Lástima -responderá Luis Hergueta a la carta de José Luis Acquaroni queriendo hacer borrón y nueva- que todos los malos entendidos que, en relación con el célebre "memorandum" que, por lo visto, nunca ha existido, y que al principio todo el mundo vió, originase tantas pegas e inconvenientes que dieron al traste con la celebración de la Bienal en la techa en la que se pensaba. Blas Piñar regresó ya de América, y viene con la promesa en firme del Ministro de Asuntos Exteriores, del de Educación, e incluso del Presidente, de celebrar en Caracas la IV Bienal Hispanoamericana de Arte. Ojalá no vuelvan a surgir los inconvenientes de la primera convocatoria y ésta pueda ser una realidad y un éxito" ".

$Y$, efectivamente, Blas Piñar regresó de Venezuela con la promesa de realización de la nueva edición del certamen en Caracas. No obstante, este mismo paso del director del ICH por el pais caribeño, parece que fue ocasión en el ambiente artístico venezolano para planear la celebración de una nueva "Bienal de Arte Latinoamericano", que parecía, en cierto modo, venir a replicar a la Bienal Hispanoamericana. La noticia había sido hecha pública a mediados de octubre por los diarios caraqueños, que daban cuenta de una rueda de prensa especialmente convocada por la Sociedad de Amigos del Museo de Bellas Artes, de Caracas, con intención de dar a conocer sus resoluciones. En ella intervinieron el director del Museo, Armando Barrios; su esposa, Reyna Rivas, y Clara Diament de Sujo, de la directiva de la Sociedad, quienes informaron de que las actividades de ésta se encauzarian en lo sucesivo "a realizar adquisiciones que propendan a la creación de una gran colección de artes plásticas latinoamericanas, con lo cual el primer museo artístico del país se convertiría también en el Primer Museo de Arte Latinoamericano". Pero además, dentro de sus iniciativas, señalaron, «la celebración en Caracas de los Festivales de Música Latinoamericana, debidos a la iniciativa privada, han hecho pensar en la realización simultánea de una "Bienal de Arte Latinoamericano", con premios y adquisiciones que estimulen el envio de obras por parte de los artistas de Latinoamérica. Es decir que nos proponemos crear una Exposición General de Artes Plásticas Latinoamericanas, cada dos años, haciendo coincidir la exposición con los Festivales de Música Latinoamericanos. Crearemos premios atractivos para los artistas y, por su parte, la Sociedad se compromete a adquirir las obras premiadas, con lo

Carta de LuIS Hergueta fechaqa: Madrid, $31-x-57$, contestanto a la de José Luis Acquaroni de techa 20-x-57 (RGAECI, Caja 2167, carp. 7199). 
cual hay doble aliciente para los concursante al par que Venezuela se beneficia con la obtención de las mejores obras» ${ }^{10}$.

No es extraño, pues, que en el Despacho con el que, a finales del mismo mes, Blas Piñar informaba al ministro español de Asuntos Exteriores de su gestiones en Venezuela, señalara que estaba "alarmado por la solapada maniobra contra la Bienal Hispanoamericana de Arte que representaría el crear, con sede en Caracas, otra Bienal de arte latinoamericano", hecho del que se habia enterado a su regreso a Madrid y que veía semejante al de la "contrabienal" de Paris surgida con la I Bienal y las “Antibienales» de La Habana con la segunda edición. Tampoco creía ajeno a ello lo positivo de sus gestiones con el Gobierno venezolano, con el que incluso habia acordado inaugurar la IV Bienal en junio de 1958, coincidiendo con la celebración de la Semana de la Patria, aunque dadas las circunstancias consideraba lo más oportuno obtener la confirmación oficial de las promesas que se le habian hecho y establecer rápidamente las condiciones de celebración del certamen. Explicaba asi estos hechos:

"Durante mi estancia en Venezuela, y como uno de los principales objetivos de mi viaje a aquella República, planteé de nuevo la celebración de la IV Bienal bajo el patrocinio del Gobierno venezolano, procurando deshacer todos los equivocos que intencionadamente habian sido creados por los escondidos enemigos que alli tiene España y empezar de nuevo estableciendo sobre firme base el futuro certamen. Me entrevisté con el Presidente de la República, con el Ministro de Educación, con el de Asuntos Exteriores y con el Ministro Secretario de la Presidencia, interesándoles directamente por la Bienal y recibiendo de todos ellos, y principalmente del Presidente de la República, la anuencia más completa. Pienso que acaso esta inesperada y repentina invención de una "Bienal de arte latinoamericano" (paralela y similar a las que se formaron en París con motivo de la 1 Bienal y en la Habana como replica a la II) es en cierto modo consecuencia de los afirmativos contactos tenidos por mi y creo que sería ocasión de salir inmediatamente al paso de este proyecto y de concretar, al mismo tiempo, la fecha de celebración de la IV Bienal Hispanoamericana.

Mi impresion es que la actitud del Embajador de Venezuela en Madrid respecto a este asunto no ha sido todo lo entusiasta que cabía esperar, ya que. según me notificó personalmente el Ministro de Asuntos Exteriores, don José Loreto Arısmendi, no había aún cursado la carta de techa 10 de mayo

Las resoluciones habian sido tomadas en sesión de la Sociedad de 14-x-57, con asistencia de Alejandro Pietri, presidente; Marcel Roche, vicepresidente; Maria Luisa Zuloaga de Tovar, subsecretaria; Juan Nouel, tesorero; Yolanda de Boulton, vocal; Carlos Rodriguez Landaeta, vocal, y Clara Diament, vocal; más los invitados Armando Barrios, Reyna Rivas y Maruja de Roche. La rueda de prensa tuvo lugar el 16-x-57. (Véase K.: "La sociedad Amigos del Museo adquirirá gran colección de arte latinoamericano", El Comercio, Caracas, 17-x-57). 
que le escribiste rogándole hiciera llegar a Su Excelencia el Presidente de la República el propósito del Instituto de Cultura Hispánica de celebrar la IV Exposición Bienal en Caracas.

En mis conversaciones con los miembros del Gobierno venezolano, y más particularmente con el Ministro de Educación Nacional y con el de Asuntos Exteriores, se acordó, en principio, inaugurar la Bienal durante la Semana de la Patria, que tendrá lugar en junio de 1958. Con ocasión de esa Semana, Venezuela va a hacer alarde de su enorme progreso material. Yo les hice notar que al Gobierno venezolano le vendría muy bien dar una nota de sensibilidad artística con la Bienal. Caracas en esos dias vendria a ser algo asi como la capital artistica del mundo hispánico.

Juzgo que lo más indicado sería seguramente solicitar cerca de nuestro Embajador en Venezuela la confirmación de las promesas que me fueron hechas y su gestión personal para lograr lo más rápidamente posible concretar las condiciones en que el certamen vaya a celebrarse" ".

Con todo, pese a las promesas reseñadas por Blas Piñar y la rápidez con la que éste quería que se procediera, la actitud oficial y venezolana volvió a hacer gala de sus vacilaciones y pasividad. De modo que era a mediados de enero de 1958 cuando el ministro de Asuntos Exteriores español recibía nota del embajador venezolano en Madrid, contestando no ya a sus últimas gestiones respecto al certamen, sino a la carta que le había dirigido en mayo del año anterior expresando el deseo de que el Gobierno de Venezuela considerará formalmente la posibilidad de que Caracas fuera sede de la IV Bienal Hispanoamericana. En respuesta a la misma, Simón Becerra transcribía la contestación del ministro de Educación de su pais, Dario Parra, quien significaba «que en la Cuenta que en el día de hoy [11-x|1-57] tuve el honor de rendir al ciudadano Presidente de la República, el Primer Magistrado de la Nación dispuso aplazar hasta el mes de enero próximo la consideración del asunto en cuestión, con objeto de realizar sobre el mismo un mejor estudio ${ }^{12}$ ".

Se trataba, en definitiva, una vez más de las características vacilaciones y dilaciones que denota toda ia actitud oficial venezolana en relación a las Bienales Hispanoamericanas. Aunque, en esta ocasión, fueron ya los mismos acontecimientos políticos internos venezolanos los que vinieron a poner el punto final a la actitud de insistencia que, por contra, tuvo ante aquella otra lo

\footnotetext{
Despacho fechado: Madrid 31-x-57 del director del ICH con el ministro de AA.EE. (AMAE, Leg. R-4511, Exp. 65).

1. Oficio $n^{\star} 761$ de fecha 11-x|1-57 del Ministro de Educación venezolano, trasladado por la nota $n^{2}$ IEB-04889 de 20-x|1-57 del Ministerio de Relaciones Exteriores venezolano al embajador de Venezuela en Madrid y, a su vez, trasladado por éste en su nota no 45 fechada: Madrid, 15-1-58 dirigida al ministro de Asuntos Exteriores español. (AME, Leg. R-5229, Exp. 67).
} 
oficial español, pues la caida del presidente venezolano Marcos Pérez Gimenez y las circunstancias políticas que se unieron en ese año de 1958 , decidieron definitivamente al Instituto de Cultura Hispánica a abandonar el proyecto de celebrar la IV Bienal Hispanoamericana en Caracas y volver la mirada hacia Quito, donde se preparaba la XI Conferencia Interamericana, coyuntura política que se ofrecia como un buen momento para llevar alli el certamen.

Las razones politicas, pues, primaron sobre las artísticas en todo el planteamiento de celebrar la IV Bienal Hispanoamericana en Caracas; ocasión que, artisticamente, acaso hubiera merecido la pena si la novedad y lo vanguardista hubieran señoreado entre los planteamientos y criterios del arte a exponer, cuestiones que, sin embargo, escasamente se examinaron y discutieron, porque de lo que aquí se habló y trató fundamentalmente no fue exactamente de arte, sino principalmente de política. La asociación con la Conferencia Interamericana, ya vieja idea meditada al paso de ésta por Caracas en 1954, y que ahora proyectaba celebrar su décimo primera edición en la capital ecuatoriana a principios de 1960, se hacía así extremadamente atractiva y una salida decente para dar cauce al fracaso venezolano.

Aunque, con todo, el desenlace de esta intención de celebrar la IV Bienal Hispanoamericana y vinculándola a las posibilidades y vaivenes de la políticas latinoamericanas, terminaría con un nuevo fracaso en Quito ${ }^{1.3}$

Resumiendo el proyecto de celebrar la IV Bienal en Quito, digamos que las negociaciones entre los gobiernos ecuatoriano y español estuvieron muy avanzadas, intentando siempre que su celebración coincidiera con la de la x 1 Conferencia Interamericana de la OEA, que la patrocinaria. Se nombró una Comisión Directiva ecuatoriana para la organización de la IV Bienal (integrada por J.G. Navarro, C.M. Larrea, Oswaldo Guayasamín, Jorge Fernández, Claudio Mena y L. Ponce Enriquez), se redactó un nuevo reglamento y se distribuyó amplia propaganda; asimismo se en. viaron las convocatorias a los distintos paises invitados - muchos de los cuales nombraron sus comisiones e hicieron su selección y se programó su fecha de inauguración para el primero de marzo de 1960 (permaneceria abierta hasta el 30 de abril del mismo año). A finales de 1959 el Consejo de la OEA postergaba la fecha de reunion de la xI Conferencia - que debia inaugurarse en Quito en el mes de febrero de 1960 - a una fecha indefinida a precisar el 30 de marzo de 1960, lo que arrastraba consigo también el aplazamiento "sine die" de la inauguración de la IV Bienal, cuya Comision comenzó a comunicar la postergacion mientras en España se devolvian a los artistas las obras seleccionas de los envios ya dispuestos a embarcar y se iniciaban gestiones para el establecimiento de la Bienal con sede fija en la península. Posteriormente, se anunció la fecha del primero de marzo de 1961 para la reunión de esta xI Conferencia, intentando el Gobierno ecuatoriano reanudar las gestiones para que a mediados del mismo mes se inaugura junto a ella la IV Bienal Hispanoamericana, pero, en general, la incertidumbe de las fechas, el descrédito, la ambigüedad de los acontecimıentos, etc. hicieron desistir de celebrarla. En este proceso, por otra parte, el proyecto de celebración en Quito tuvo también sus actitudes "antibienal", especialmente entre los artistas de Mexico, Venezuela, Perú y notablemente en Cuba, pese a que asirnismo se hubieran conseguido ya algunos Iogros de otro caracter, como el premio ofrecido por la UNESCO a los participantes o la solicitud de Nueva Orleans de llevar alli una antológica de esta IV Bienal tras su clausura en Quito. 
que acabaría por poner fin a la historia de las Bienales Hispanoamericanas de Arte. Final que, de forma efectiva les llegaba a comienzos de los años sesenta, ya en los momentos de dirección del Instituto de Cultural Hispánica por Gregorio Marañón, - de hecho hasta entonces tuvo vida la Secretaria Permanente de la Bienal Hispanoamericana de Arte que funcionaba en el seno del $\mathrm{ICH}$ y que dirigió, hasta su muerte en agosto de 1962, el poeta Leopoldo Panero-, en los que las Bienales se transformaron o, mejor, fueron sustituidas, especialmente por consejo de Luis González Robles, hasta entonces vicesecretario general de la Bienal, por las exposiciones - sin el adjetivo de bienal, que comprometía más la continuidad- de "Arte de América y España" que organizó el propio González Robles y cuya primera edición, inaugurada en 1963 en Madrid, no sólo mostró ya el final de las Bienales y el giro producido en una determinada vía de política artistica española, sino que, además, a la vez indicó el comienzo de un nuevo momento en el arte español ${ }^{14}$.

Tras ano y medio de preparativos, la primera edición fue inaugurada en los Palacios de Cristal y Velázquez del Retiro madrileño en mayo de 1963 y posteriormente se traslado a Barcelona y otras ciudades (Salamanca, Sevilla, Valencia, San Sebastian, Bilbao, Lisboa, Berlin. Napoles, Roma y Berna). Participaron 43 artistas españoles junto a los de otros 26 paises, entre ellos EE.UU. y Canada, conformando un conjunto de 600 obras que, si por un lado, recogia al informalismo - pese a ausencias, por discrepancias, como las de Tápies, Millares o Saura-, por otro, se abría a nuevas tendencias como el pop. con la presencia de algunos de sus mayores re. presentantes a nivel internacional -Rauschenberg, Jasper Johnes, Larry Rivers-. Aparte, pues, de la pretension de acreditación y prestigio de los nuevos derroteros y vias de actuación de la estera oficial espanola en el campo del arte contemporáneo, la muestra significó, a la vez que una síritesis del periodo de predominio informalista, un apunto de las nuevas tendencias que iban a encontrar su pujanza en los años siguientes, es decir, la exposición vino a poner de manifiesto la aparición de la opción neofigurativa frente a la informalista. (Véase Arte de América y España. Catálogo General. Madrid. Mayo-Junio 1963. Barcelona, Agosto-Septiembre 1963, Madrid, Instituto de Cultura Hispanica-Gráficas Reunidas, 1963; sobre su recorrido por Europa: "La Exposición de Arte de America y España". Artes, $n^{\circ}$ 51, Madrid, 8-11-64, pág. 5; sobre su organización: Luis Gonzalez Robles: "Mis recuerdos de aquella década", en el catálogo: Madrid. El arte de los 60. Madrid, Sala de Exposiciones de la Comunidad, 1990, págs. 23-29). 
